\title{
The COPD criteria
}

Malcolm Campbell was a member of the expert group which advised the negotiators (NHS Confederation and General Practice Committee) on the criteria for the Quality Outcomes Framework (QOF). This group has since been disbanded and the following comments constitute Dr Campbell's personal opinion.

It is important to realise that the United Kingdom clinical Quality Outcomes Framework is not a set of guidelines, nor is it a diagnostic classification. It is a method of providing financial rewards to Practices for providing clinical care. UK general-practices are rewarded financiatly if theyattain points under this system. There are 72 points avaitable for asthma and 45 available for Chronic Obstructive Pulmonary Disease (COPD); each point is worth $£ 75$ this year and $£ 125$ next year, proportionally adjusted according to total list size and disease prevalence compared to the national average.

Our overall objective in advising both sets of negotiators (The General Practice Committee and the NHS Confederation) was to recommend criteria which would result in better patient care. In general terms the indicators had to be recordable consistently on an IT database (in this case being transferred from the primary care computer system onto the Department of Health assessment system (QMAS)) in order to allow reporting and monitoring to take place. Also, we needed to have a workable number of indicators for each clinical area which did not over-burden the clinician and which, if measured, would ultimately improve patient care.

In the case of asthma, for example, we used the concept of 'active asthma'. Active asthma was defined as a previous Read-coded diagnosis of asthma AND a prescription for anti-asthma medication in the previous 12 months. We recom- mended excluding patients, for the purposes of payment, who were recorded as having asthma but whose disease was inactive. We assumed that if the patient required no treatment, their asthma was unlikely to be causing significant symptoms, and therefore any effort put into monitoring would result in minimal benefit to the individual. We also assumed that these patients would be unlikely to turn up for review.

In the case of COPD we reviewed the available guidelines [1,2] and identified quality markers which had an evidence base and which would be cost-effective. As a result the criteria we set emphasise spirometric diagnosis, smoking cessation advice, regular review and therapeutic trials of inhaled steroids and anti-cholinergic drugs.

Our decision to recommend that, for the purposes of payment, the upper level of FEV1 should be $70 \%$ predicted rather than the $80 \%$ in the GOLD [1] guidelines has been criticised in the UK medical press. We were careful to point out that the $70 \%$ level chosen was different from the GOLD definition. Health professionals should realise that COPD does not begin when FEV1 reaches $80 \%$ or $70 \%$. Any cut-off point is by definition arbitrary. We selected $70 \%$ because we assumed that this would include most symptomatic patients where intervention would be most effective. The new General Medical Services (nGMS) contract already rewards practices for promoting smoking cessation in all patients. We took the view that any additional activity required in the $70 \%$-to- $80 \%$ group would be minimal other than recommending smoking cessation, which is already rewarded in the QOF, and would place a huge strain on the limited spirometry services available. Studies carried out on patients with asymptomatic COPD have shown conflicting 
results in terms of any increased tendency to quit smoking, based on patient knowledge of the presence of early COPD.

The overall objective of the QOF was to produce the greatest benefit for the greatest number of patients. It was our view at the time that restricting the QOF to patients likely to be symptomatic would produce the most effective results. The QOF will be reviewed in 2006 and, no doubt, careful consideration will be given to any suggested changes.

\section{References}

[1] National Institutes of Health NHLaBI. Global Initiative for Chronic Obstructive Lung Disease. Global Strategy for the
Diagnosis, Management, and Prevention of Chronic Obstructive Pulmonary Disease. http://www.goldcopd.com/. 2003. Last Accessed on 9-1-2004.

[2] BTS/SIGN. British guidelines on the management of asthma. Thorax 58[Suppl 1]www.sign.ac.uk, 1-94. 2003. http://www.sign.ac.uk/guidelines/fulltext/63/update2004. html.

Malcolm Campbell University of Glasgow Southbank Surgery, Kirkintilloch Glasgow G66 1NH, UK Tel.: +44 1417762183

E-mail address: malcolm.campbell@virgin.net

19 November 2004

Available online at www.sciencedirect.com

science $\boldsymbol{d}$ Directo

Available online at http://www.thepcrj.com

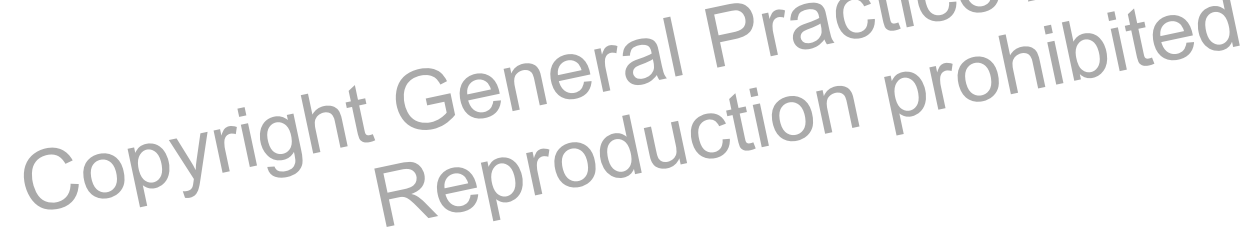

